\title{
Formation of Magnetic Textures in the Ferromagnetic Phase of $\mathrm{La}_{0.825} \mathrm{Sr}_{0.175} \mathrm{MnO}_{3}$
}

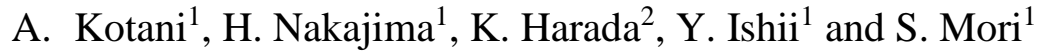 \\ 1. Department of Materials Science, Osaka Prefecture University, Sakai, Osaka, Japan. \\ 2. RIKEN Center for Emergent Matter Science (CEMS), Wako, Saitama, Japan.
}

Magnetic textures such as magnetic bubbles and magnetic stripe domains have been attracted attention in some magnets because of their potential practical applications to new spin devices and quantum computing applications ${ }^{1}$. Recently it was found that magnetic textures appeared in some chiral magnets such as FeGe without the inversion symmetry by neutron scattering experiment and Lorentz microscopy observation $^{2,3}$. On the other hand, it was reported that some compounds with perovskite-related structures having the inversion symmetry such as $\mathrm{La}_{0.875} \mathrm{Sr}_{0.125} \mathrm{MnO}_{3}$ exhibited magnetic textures such as magnetic bubbles in the ferromagnetic phase ${ }^{4}$. Complex magnetic textures at room temperature were also reported in amorphous ferromagnetic Fe/Gd films with perpendicular magnetic anisotropy ${ }^{5}$. Based on these experimental results, it is anticipated that magnetic textures such as magnetic bubbles should appear in the ferromagnetic phase with the inversion symmetry. Thus, we have investigated magnetic textures in the ferromagnetic metallic phase of $\mathrm{La}_{0.825} \mathrm{Sr}_{0.175} \mathrm{MnO}_{3}$ with the inversion symmetry by smallangle electron diffraction (SmAED) and Lorentz microscopy experiments.

Single crystals of $\mathrm{La}_{1-x} \mathrm{Sr}_{x} \mathrm{MnO}_{3}(x=0.175)$ were grown by the Floating Zone method. A thin plate for the Lorentz microscopy observation was prepared by grinding with alumina powder and subsequently $\mathrm{Ar}^{+}$ion sputtering at room temperature. An in-situ Lorentz microscopy observation was carried out in order to elucidate magnetic textures and magnetic responses of magnetic textures by applying magnetic field perpendicular to the thin plate. Note that external magnetic field was applied to the thin plate by exciting objective lens of the transmission electron microscopy (TEM).

Magnetic stripe domains in the orthorhombic structure with the inversion symmetry of $\mathrm{La}_{0.825} \mathrm{Sr}_{0.175} \mathrm{MnO}_{3}$ has been reported in our previous work ${ }^{6}$. Thus, in this work we investigated changes of magnetic stripe domains by applying external magnetic field. Figure 1 displays changes of magnetic stripe domains by applying external magnetic field perpendicular to the (111) plane at $100 \mathrm{~K}$ as a function of the strength of external magnetic field. Magnetic stripe domains with magnetic moments antiparallel between adjacent magnetic domains, which was characterized as the $180^{\circ}$ magnetic domains, were formed in the orthorhombic structure without external magnetic field. As the external magnetic field was applied perpendicular to the (111) plane, it was found that the magnetic stripe domains transformed into fragmentary magnetic domains with the elliptical shape, as shown in Fig. 1(b), and finally disappeared at $B=100 \mathrm{mT}$. Spatial distribution of the magnetic moments inside the fragmentary magnetic domain is depicted in Fig.1(c). As the strength of the magnetic field was decreased from $B=$ $100 \mathrm{mT}$, the magnetic stripe domains appeared around $B=50 \mathrm{mT}$ reversibly with the thermal hysteresis. On the other hand, we found the transformation from magnetic stripe domains to magnetic bubbles by applying external magnetic fields perpendicular to the (001) plane at $100 \mathrm{~K}$. Note that the [001] direction is parallel to the magnetic easy axis in the orthorhombic structure. As shown in Fig. 2(a), meandering magnetic stripe domains with alternative bright and dark contrasts can be seen clearly in $B=$ $0 \mathrm{~T}$. Note that red arrows in Fig. 2(a) represent the directions of the magnetic moments. When the magnetic field was applied perpendicular to the thin plate, type-I magnetic bubbles with the counterclockwise and clockwise curl of the in-plane magnetization appeared, as shown in Fig. 2(b). In 
addition, type-II magnetic bubbles are also distributed in a random manner. That is, three distinct types of magnetic bubbles can be observed, as shown in three dotted squares of Fig. 2(b). Figure 2(c) shows illustrated description of these three distinct types of magnetic bubbles; (1) type-I magnetic bubbles with counterclockwise spin helicity, (2) type-I magnetic bubbles with clockwise spin helicity and (3) type-II magnetic bubbles. It is revealed that the appearance of the complex magnetic textures of type-I and typeII magnetic bubbles by applying external magnetic field should depend on the magnetic anisotropy. [7]

\section{References:}

[1] N. Nagaosa and Y. Tokura, Nature Nanotech. 8899 (2013).

[2] S. Mühlbauer, et al., Science 323, 915 (2009).

[3] X. Z. Yu, et al., Nature 465, 901 (2010).

[4] T. Nagai, et al., Appl. Phys. Lett. 101, 162401 (2012).

[5] J. Chess et al., Microsc. Microanal. 21 (Suppl 3), 0823 (2015).

[6] T. Asaka et al., J. Mag. Mag. Mat. 310, 782-784 (2007).

[7] This work was partially supported by a Grant-in-Aid for Scientific Research from the Ministry of Education, Culture, Sports, Science and Technology of Japan (MEXT) (No. 15K13306).

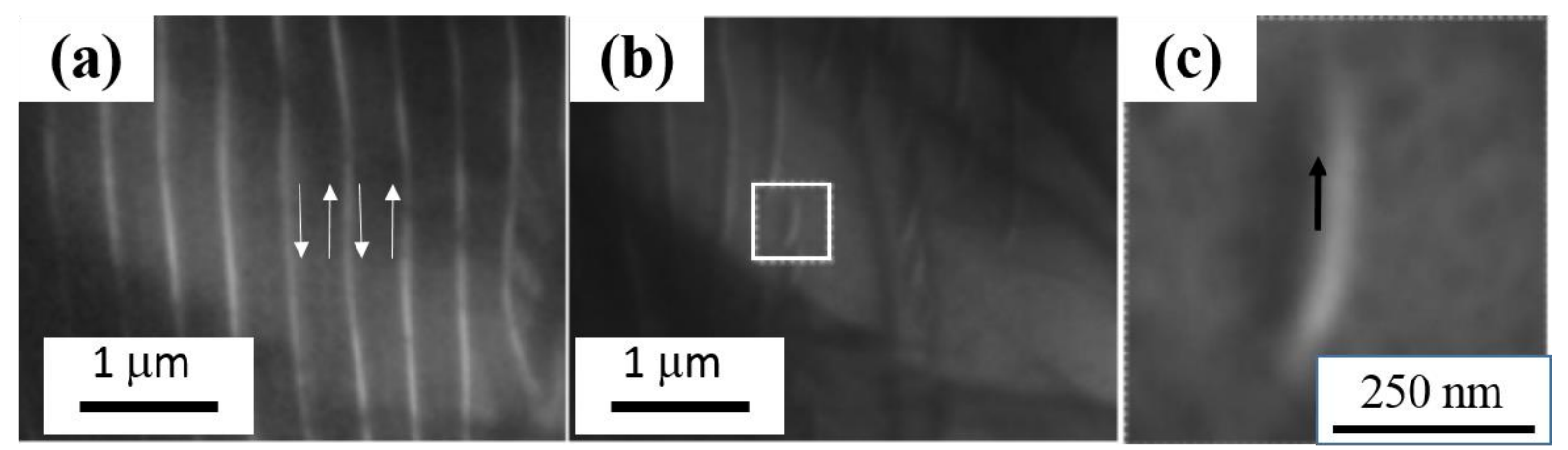

Figure 1. Changes of magnetic stripe domains in the (111) crystal plane as a function of the strength of magnetic field normal to the thin film of $\mathrm{La}_{0.825} \mathrm{Sr}_{0.175} \mathrm{MnO}_{3}$. The strengths of the magnetic field were (a) 20 $\mathrm{mT}$ and (b) $100 \mathrm{mT}$, respectively. (c) Magnified fragmentary magnetic domain with the elliptical shape was shown, together with the direction of magnetic moment in the fragmentary magnetic domain.
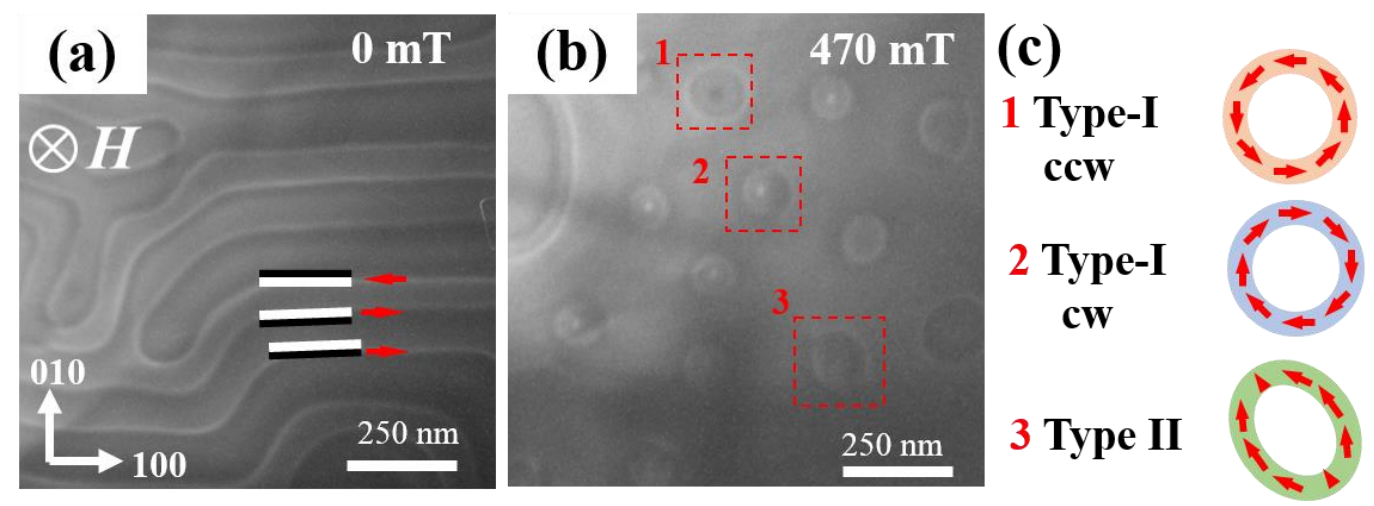

Figure 2. Variation of magntic domain structures as a function of the strengths of external magnetic field. The strengths of the magnetic field are (a) $0 \mathrm{~T}$ and (b) $470 \mathrm{mT}$, respectively. (c) Schematic description of three distict types of magnetic bubbles found in (b). Arrows in (a) and (c) represent the direction of the magnetic moments. 